\title{
Repair of $P$ element ends following hybrid element excision leads to recombination in Drosophila melanogaster
}

\author{
$X$ Liang and JA Sved \\ School of Biological Sciences A12, University of Sydney, New South Wales, Australia
}

\begin{abstract}
$P$ elements are thought to replicate themselves starting with the association of the left and right ends, followed by a cutcopy-paste process. An abnormal form of this process has been shown to occur when the associated left and right ends come from sister elements rather than from the same element, leading to formation of a 'hybrid element.' These ends can insert nearby in the genome to produce recombination, with associated structural changes. We have previously increased the frequency of such 'hybrid element insertion' by combining end-deleted elements in trans in a genotype with a left-end on one chromosome and a right-end on the homologous chromosome. Although many recombinants produced by this genotype have structural changes
\end{abstract}

expected with insertion, nearly $50 \%$ of the predicted insertional recombinants contain no structural change. We present evidence using RFLP markers closely linked to the end-deleted elements that in these cases the $P$ element ends dissociate before insertion, and are subsequently ligated together following a process analogous to synthesis-dependent strand annealing. The results suggest that broken ends containing $P$ elements are resolved by the same repair process as ends not containing $P$ elements, and that such repair from hybrid element events may occur in the majority of cases.

Heredity (2009) 102, 127-132; doi:10.1038/hdy.2008.87; published online 10 September 2008

Keywords: Drosophila melanogaster, $P$ elements; recombination; hybrid elements; DNA repair; SDSA

\section{Introduction}

Engels et al. (1990) introduced the cut-copy-paste model for the propagation of $P$ elements and possibly other transposons. Under this model, $P$ element mobility is initiated by an excision event (see the left-hand side of Figure 1). Two separate events then ensue. First, the element inserts elsewhere in the genome. Second, the double-strand gap left by the excision event is repaired. Providing that the excision event occurs at the fourstrand stage, and that repair primarily uses the sister strand as a template, the repair process will restore an exact copy of the element at the original site.

Evidence for the model is indirect, coming largely from the minority of cases where the repair process uses either a non-sister or non-homologous copy of the excision site. As sister-strand repair leaves no fingerprints, there is no direct evidence for this part of the process. However, the model is in accord with many aspects of $P$ element behavior and, in particular, explains how a single 'transposase' protein (O'Hare and Rubin, 1983; Beall and Rio, 1997) could account for all the processes needed for the increase in copy number of an element. A primary requirement for the process is for excision to occur at the

Correspondence: Dr JA Sved, School of Biological Sciences A12, University of Sydney, Biology A12, New South Wales 2006, Australia. E-mail: jsved@usyd.edu.au

Received 5 March 2007; revised 4 March 2008; accepted 27 July 2008; published online 10 September 2008 four-strand stage, so that the restoration of the original element is achieved purely by the host's repair machinery. It has not, however, been possible to demonstrate a preference for excision at the four-strand stage in a tissue culture system (Weinert et al., 2005).

The cut-copy-paste model provides the basis for an explanation of how $P$ elements cause chromosome breakage and recombination (Gray et al., 1996; Preston and Engels, 1996; Preston et al., 1996). These authors postulated that in a fraction of cases, the left- and righthand ends that associate with initiate excision come not from the same element but from sister elements (see the right-hand side of Figure 1). This leads to the formation of a 'hybrid element', and insertion of this element elsewhere in the genome leads to a recombination event.

The evidence presented by Gray et al. (1996) relied on the production of a male genotype containing two enddeleted elements, one missing the left-end and the other missing the right-end (Figure 2a). The only ends available for pairing in this case come from homologous chromosomes (Figure 2b), rather than sister elements as in Figure 1. The 'excision' event then leads to elementcontaining ends $e_{1}$ and $e_{2}$ available for insertion and ends $n_{1}$ and $n_{2}$ available for repair (Figure $2 c$ ).

Three potential sites of insertion of the $e_{1}$ and $e_{2}$ hybrid element are shown by asterisks in Figure 2c. These three are expected to lead to recombinant chromosomes as shown in Figure 2d. The first of these, site 1, contains a deletion to the left of the original insertion point, whereas site 2 contains an insertion of a portion of the 


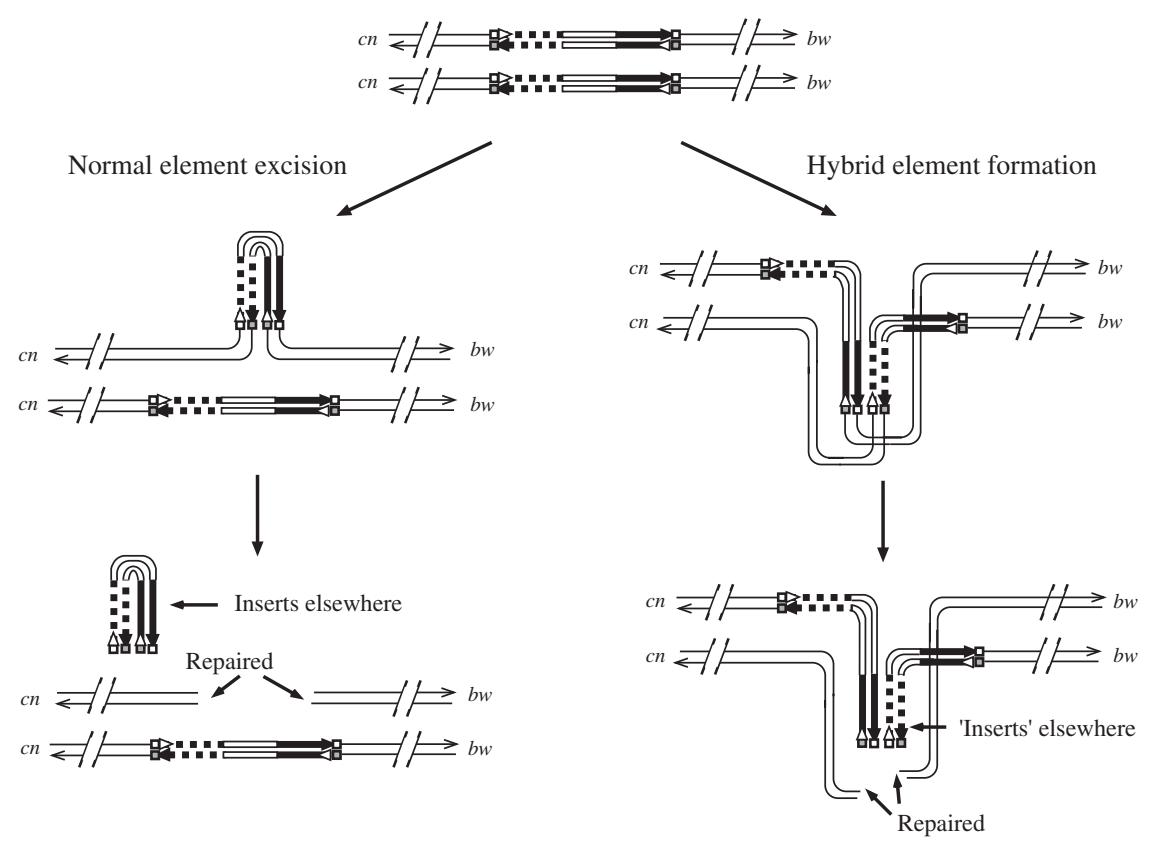

Figure $1 P$ elements shown at the four-strand stage (top of figure). The two ends of the element are shaded differently and the doublestranded nature of DNA is shown for compatibility with models introduced later (see Discussion). The remainder of the figure shows the processes of normal $P$ element excision (left side) and abnormal excision through pairing of sister $P$ element ends to form a hybrid element (right side). For simplicity, the diagram shows normal excision as leading to a free element, although in reality there may be an association with the point of insertion at all stages in both processes.

a

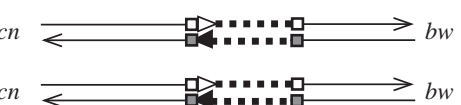

$c n$

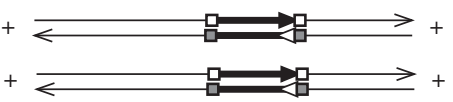

d

(1)

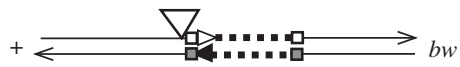

(2)

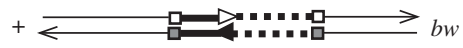

(3) $+\underset{\mathrm{a}}{\rightleftarrows} \longrightarrow \mathrm{bw}$
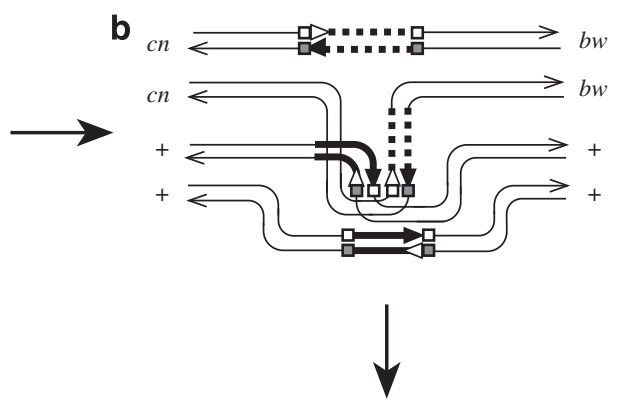

C

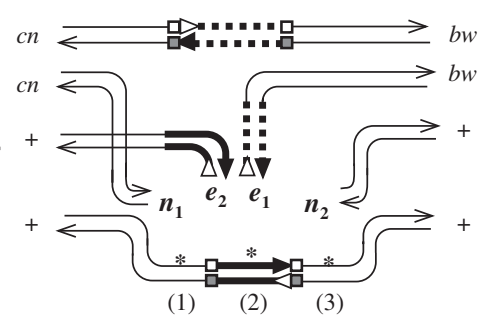

Figure 2 Hybrid element formation in a genotype containing opposite end-deleted elements. The original genotypes at the four-strand stage are shown in (a). (b) Shows the way in which opposite ends can associate to form a hybrid element. (c) Shows the consequences of 'excision' of the hybrid element, leading to element-containing ends $e_{1}$ and $e_{2}$ and ends lacking an element, $n_{1}$ and $n_{2}$. (d) Shows three possible recombinant genotypes arising from insertion into three sites marked with asterisks in (c).

element and site 3 contains an insertion of a complete element end plus chromosomal DNA. The recombination events can be detected through the use of outside markers $c n$ and $b w$.

Note that the three insertion sites and the recombinant chromosomes shown in Figures 2c and d constitute only a fraction of the possible events (see Gray et al., 1996; Figure 2). However, the recombinant chromosomes all share the property that those of genotype $+b w$ are expected to arise through insertion of the $e_{1}$ and $e_{2}$ hybrid element, whereas those of genotype $c n+$ are expected to arise through repair of the $n_{1}$ and $n_{2}$ ends. Gray et al. introduced the terminology of HEI (hybrid element insertion) to describe the $+b w$ insertion events and HER (hybrid excision and repair) to describe the $c n+$ repair events.

Gray et al. (1996) found that $15 \%$ or more of offspring from the genotypes shown in Figure 2 were $+b w$ recombinants, with slightly more of $c n+$ recombinants. The difference in frequency between these reciprocal types could be accounted for in terms of the inviability of 
some of the expected insertion genotypes. Many such genotypes are expected to lead to dicentric bridges and fragments (Figure 2).

One unresolved feature of the results of Gray et al. was the finding of a large number, approximately $50 \%$, of $+b w$ recombinants containing no chromosome structural changes. It can be seen from Figures $2 \mathrm{c}$ and $\mathrm{d}$ that unless the hybrid element inserts precisely at the element ends, all HEI recombinants should contain either deletions or else insertions with part or all of both elements. This suggests that HEI might not be the only way of resolving hybrid elements to produce recombination. Simple symmetry shown in Figure 2 would suggest that the repair processes governing the repair of the $n_{1}$ and $n_{2}$ ends might also apply to the $e_{1}$ and $e_{2}$ ends. This would require the dissociation of element ends before insertion, a process that is considered in more detail in the Discussion section.

The purpose of this paper, then, is to examine the possibility of repair of $P$ element-containing ends. This has been carried out through the use of RFLP markers closely linked to the $P$ element ends, allowing for the first time an analysis of the products of recombination in the region of the $P$ element.

\section{Materials and methods}

Stocks and procedures used in this paper are mostly as outlined in Svoboda et al. (1995). All results in this paper are from crosses involving deleted elements derived from a single initial insertion of the $P[\mathrm{CaSpeR}]$ transposon in the 50C region of chromosome 2 . These deletions were produced under the action of the transposase source $P(\Delta 2-3)(99 \mathrm{~B})$. The deleted elements have subsequently been maintained in stocks in the absence of the transposase source. The two elements involved in the current experiment are a left-end element (that is, one having a deletion of the right-end), labeled as DL1 and a right-end element labeled as DR2.

The primary aim of the experiment reported here was to set up a cross in which males are heterozygous for the left-end element DL1 and the right-end element DR2, together with distant flanking markers $c n$ and $b w$ and the transposase source $P(\Delta 2-3)(99 B)$ (see Svoboda et al. 1995, Figure $5 \mathrm{a}$ ). In addition, the immediate region of $50 \mathrm{C}$ containing the $P[\mathrm{CaSpeR}]$ insertion was marked with RFLPs or insertions (Figure 3). Four such markers, two

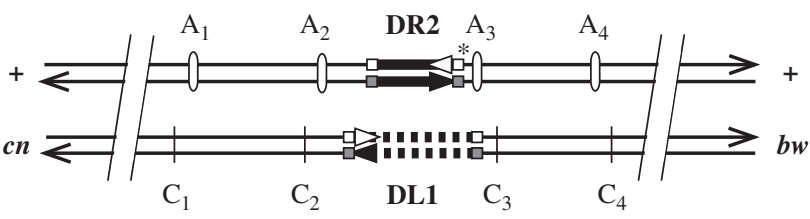

Figure 3 Genotype for analysis of recombination events in the region of $P[C a S p e R](50 \mathrm{C}) . A_{1}: R S a \mathrm{I}$ site, $2.5 \mathrm{~kb}$ to the left of insertion site; $A_{2}: M S p I$ site, $500 \mathrm{bp}$ to the left of insertion site; $A_{3}$ : EarI site, $30 \mathrm{bp}$ to the right of insertion site; $A_{4}: 300 \mathrm{bp}$ deletion, $1.4 \mathrm{~kb}$ to the right of insertion site. The markers shown with unfilled ovals are denoted as $A$, with the wild-type chromosome markers denoted as $C$ (Preston and Engels, 1996). The asterisk indicates the position of a $21 \mathrm{bp}$ deletion in the DR2 $\left(A_{2}\right)$ stock (see text). Although the positions of the RFLP markers are not drawn to scale, the $A 3 / C 3$ marker is, as indicated, closer to the elements than the $A 2 / C 2$ marker. on either side of the element, were used in the current experiment. These markers were detected using four primer pairs surrounding the markers (Preston and Engels, 1996). Markers $A_{1}, A_{2}$ and $A_{3}$ were detected using enzyme digests of the PCR fragments, whereas $A_{4}$ was detected using fragment size. Around 200 of each of the $+b w$ and $c n+$ recombinant progeny were selected for analysis.

To produce the end-deleted element surrounded by the $A$ RFLP markers shown in Figure 3, we started with stocks kindly provided by Christine Preston, containing the full-length $P[\mathrm{CaSpeR}](50 \mathrm{C})$ element surrounded by the $A$ markers. A male genotype was constructed containing a chromosome of this genotype opposite to the right-end deleted element $D R 2$, plus the transposase source $P[\Delta 2-3](99 \mathrm{~B})$. This genotype produced many progeny in which the full-length element had excised and been replaced by the end-deleted element (Svoboda et al., 1995). Among these, some chromosomes were shown using PCR and FRLP digestion to contain the required four flanking markers. The procedure was carried out using two different $A$-containing stocks, one labeled $A 1$ that was chromosomally normal, and the second, $A 2$, that was shown to contain a $26 \mathrm{bp}$ deletion immediately to the right of the $P[\mathrm{CaSpeR}](50 \mathrm{C})$ element.

\section{Results}

Table 1 summarizes the recombination results from the genotypes shown in Figure 3. The first two lines are genotypes containing the RFLP markers, the $A_{1}$ stock and the $A_{2}$ stock, containing the additional $23 \mathrm{bp}$ deletion. The third line shows a control cross in the absence of the RFLP markers. As shown by the comparison of lines 1 and 3, the RFLP markers have no effect on the rate of recombination (24.9\% recombination versus $24.5 \%$ ). However, the presence of the deletion in the region of the element significantly reduces the rate of recombination (10.9 versus $24.5 \%$ ).

Table 2 summarizes analysis of recombinant chromosomes for the RFLP markers. The table shows both classes of recombinants, $+b w$ and $c n+$, and is further subdivided according to whether the progeny contain the left element DL1 or the right element DR2. The class of element contained by the progeny was determined using the four-primer PCR procedure outlined by Svoboda et al. (1995). Many progeny, mostly among the $+b w$ recombinants, were also shown to contain novel bands, indicative of structural chromosome changes. These structurally altered chromosomes, consistent with the HEI model (Gray et al., 1996), were eliminated from consideration in the present experiment.

The principal interest in the table is in the analysis of the $+b w$ offspring, as these are expected to result from resolution of $P$ element-containing ends. The results are

Table 1 Recombination results from end-deleted $P$ elements with and without RFLP markers

\begin{tabular}{lccrrrr}
\hline Male genotype & $c n$ bw & ++ & $c n+$ & $+b w$ & Total & Rec\% \\
\hline DL1/DR2 (A1) & 4075 & 4438 & 1436 & 1384 & 11333 & 24.9 \\
DL1/DR2 (A2) & 5855 & 5701 & 598 & 756 & 12910 & 10.5 \\
DL1/DR2 (C) & 2011 & 2269 & 654 & 733 & 5667 & 24.5 \\
\hline
\end{tabular}


not significantly different for the $D R 2\left(A_{1}\right)$ and $D R 2\left(A_{2}\right)$ elements and have been combined in the final column. Only a few $c n+$ recombinants were analyzed for the $D R 2\left(A_{2}\right)$ element, and these have been omitted for simplicity, as they did not show any differences from the $D R 2\left(A_{1}\right)$ results.

\section{Discussion}

Several aspects of the results in Table 2 are of interest. First, the majority of progeny, 344/360, showed recombination between the outer RFLP markers. The exceptions to this, in which the RFLP markers and elements

Table 2 Flanking marker, element and RFLP genotypes of progeny from parental genotype +A A DR2 A A+/cn C C DL1 C C bw

\begin{tabular}{|c|c|c|c|c|c|}
\hline \multirow{2}{*}{$\begin{array}{l}\text { Recombinant } \\
\text { type }\end{array}$} & \multirow{2}{*}{$\begin{array}{l}\text { Element } \\
\text { genotype }\end{array}$} & \multirow[t]{2}{*}{ RFLP type } & \multicolumn{3}{|c|}{ Parent } \\
\hline & & & $A 1$ & $A 2$ & Total \\
\hline \multirow[t]{7}{*}{$+b w$} & \multirow[t]{3}{*}{ DL1 } & A A.C C & 70 & 58 & $128(2)$ \\
\hline & & A C.C C & 11 & 4 & 15 \\
\hline & & $\mathrm{C} \mathrm{C} . \mathrm{C} \mathrm{C}$ & - & 1 & 1 (1) \\
\hline & \multirow[t]{4}{*}{ DR2 } & A A. A C & 19 & 15 & $34(3)$ \\
\hline & & A A. A A & 7 & 7 & $14(1)$ \\
\hline & & A C. A A & 1 & - & $1(4)$ \\
\hline & & A A. C C & 3 & - & 3 \\
\hline \multirow[t]{6}{*}{$c n+$} & \multirow[t]{3}{*}{ DL1 } & C C.C A & 25 & - & $25(3)$ \\
\hline & & C C. A A & 10 & - & 10 \\
\hline & & $\mathrm{CC} . \mathrm{C} \mathrm{C}$ & 1 & - & 1 (1) \\
\hline & \multirow[t]{3}{*}{ DR2 } & C C. A A & 120 & - & $120(2)$ \\
\hline & & C A.A A & 6 & - & 6 \\
\hline & & C C.CA & 2 & - & $2(5)$ \\
\hline
\end{tabular}

See text for meaning of descriptors (1)-(5). are one or other parental type, are marked as (1) in Table 2. Although the HEI and hybrid excision and repair model predicts that most recombination events will occur in the immediate region of the element (Gray et al., 1996), this has not previously been directly demonstrated for end-deleted recombination.

The second point of interest concerns the joint segregation of the proximal marker pairs $A 2 / C 2$ and $A 3 / C 3$ with the element. Where the recombination point lies to the left of the element, recombination is primarily in the region between $A 2 / C 2$ and the element (see lines labeled (2) in Table 2). In contrast, when the recombination point lies to the right of the element, it is primarily in the region between $\mathrm{A} 3 / \mathrm{C} 3$ and $\mathrm{A} 4 / \mathrm{C} 4$ markers, that is, the $\mathrm{A} 3 / \mathrm{C} 3$ markers largely segregated with the element (see lines labeled (3)). This difference is consistent with the fact that the A3/C3 marker pair lies only $30 \mathrm{bp}$ from the element, whereas the A2/C2 marker pair lies $500 \mathrm{bp}$ distant.

Perhaps the point of greatest interest concerns the less frequent classes and the predictions from repair models. We consider here the synthesis-dependent strand-annealing (SDSA) model. This model satisfactorily accounts for the precise repair of double-stranded breaks on a single chromatid using just DNA synthesis and ligation (Nassif et al., 1994; McVey et al., 2004; Preston et al., 2006). Here it is applied to the joining of two broken chromatids that were previously separate, but the principle is the same. Figure 4 shows the model considered separately for ends containing elements $\left(e_{1}\right.$ and $e_{2}$ leading to $+b w$ recombinants-left side) and ends not containing elements $\left(n_{1}\right.$ and $n_{2}$ leading to $c n+$ recombinants-right side).

Synthesis occurs on one DNA strand against the homologous strand on the sister chromatid. For example, in Figure 4, extension of one strand of chromatid (2) on
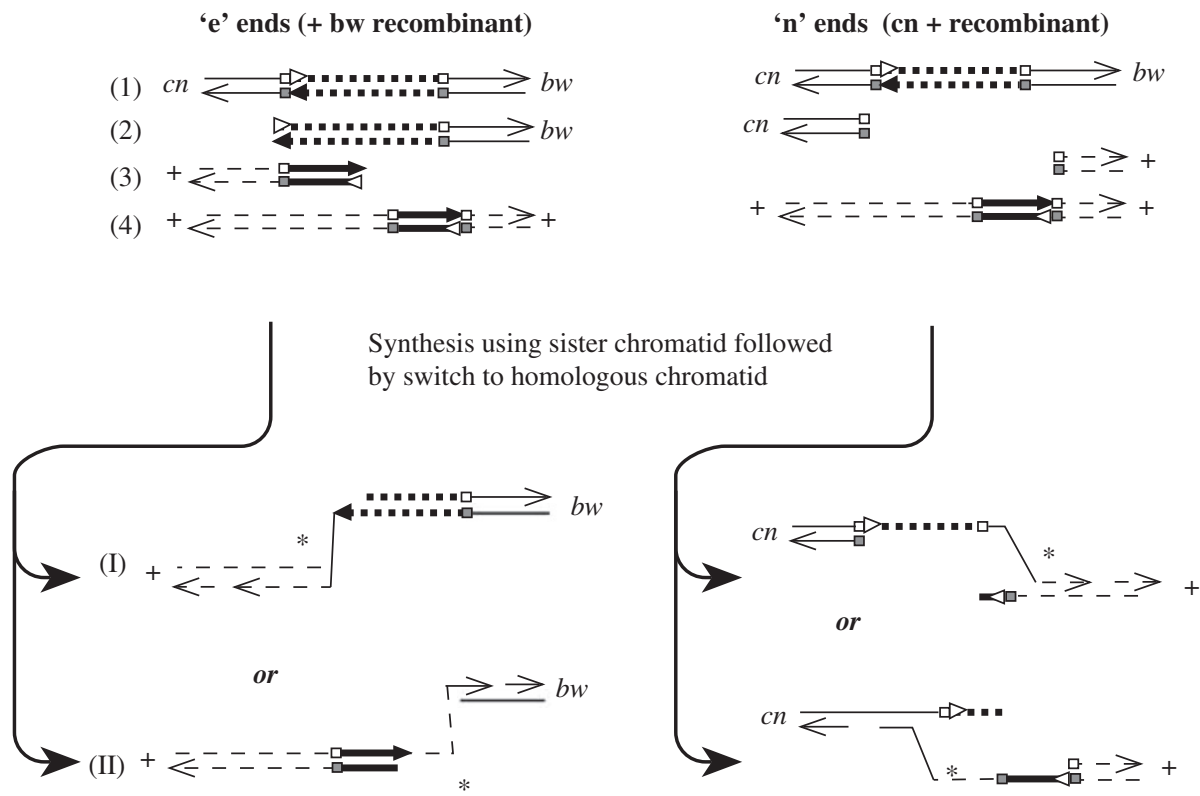

Figure 4 The synthesis-dependent strand-annealing (SDSA) model with unidirectional synthesis, showing chromatid ends after excision (cf. (c) of Figure 2), followed by an intermediate stage after pairing is established with the homologous strand but before the joining process is completed by ligation and repair. The $A$ strand is shown using a dashed line and the $C$ strand using an unbroken line. The original chromatids on the left side are labeled (1)-(4) (see text) and the two possible recombinant chromosomes as (I) and (II). Asterisks mark the end of the element at which repair is expected under the model. 
the left side of the figure proceeds in a $5^{\prime}-3^{\prime}$ direction, copying from the complementary strand of (1), leading to outcome (I). Once the element end and some of the flanking region has been copied, synthesis can then switch to the homologous strand of chromatid (3), displacing the equivalent strand. This needs to be followed by back synthesis of the complementary strand of chromatid (3) to repair the single-stranded gap.

Where different strands are to be joined in a double helix, the pairing of non-sister chromatids will involve mis-matched helices at polymorphic bases. The mismatches need to be resolved by a second round of repair, leading to conversion tracts. Importantly, in the present case, provided that the process is unidirectional as pictured in Figure 4, these tracts are expected to occur only at the $3^{\prime}$ end of the synthesis, marked by asterisks.

At the same time as strand (2) is being synthesized using strand (1), strand (3) is expected to synthesize against strand (4), leading to outcome (II). Synthesis presumably starts on both strands (2) and (3), but homology needs to be established only on one of the two newly synthesized strands, following which the joining process can be completed by degradation of the newly synthesized material on the strand that has not established homology. Occasionally, however, homology would be expected to establish on both newly synthesized strands, in which case the process would be bidirectional and conversion tracts could form at both ends (Nassif et al., 1994).

A principal prediction from the SDSA model may be summarized as follows. For the $+b w$ recombinants, there should be no recombination between the incomplete end of the element and the proximal RFLP marker. Conversely, for the $c n+$ recombinants, there should be no recombination between the complete end of the element and the proximal marker. Among the 360 recombinants analyzed in Table 2, there are only three exceptions to this prediction. One of these, labeled (4), involves a $+b w$ recombinant, and two, labeled (5), involve $c n+$ recombinants. As argued above, these exceptions can be explained if bidirectional SDSA occurs in a small percentage of cases.

Another aspect is the previously described co-segregation of the DR2 element with the right-side $A 3$ marker and DL1 with C3 marker (Figure 3), compared to the opposite result for the left side, where the A2/C2 markers segregate largely with the outside $\mathrm{cn} /+$ markers rather than with the element. Under the model of Figure 4, this discrepancy is expected simply because it is unlikely that switching of the synthesis to the opposite strand will occur in the short distance between the element and A3/C3 (30 bp), compared to the longer distance between A2/C2 and the element (500 bp).

One further aspect of the results concerns the relative numbers of the two classes of elements found in recombinant chromosomes. The large discrepancy between the two elements in the $c n+$ recombinants, 38:130, can be explained by the fact that the right-end element is much smaller than the left-end element, so that homology is likely to be established first after synthesis of the small element (Gray et al., 1996). On the other hand, the excess of the larger left-end element in the $+b w$ class, 144:51, cannot be explained in the same way. The situation is complicated here because it is not clear how much of the element is degraded before synthesis against the sister chromatid begins. The excess of the large element suggests that the element is largely not degraded, which may lead to a greater ease of establishing homology in this case.

\section{Conclusions}

$P$ element-induced recombination has largely been accounted for by a mixture of HEI and hybrid excision and repair events (Gray et al., 1996). The one result at variance with this model, the result that initiated this study, was the observation of a large number of what ought to be insertional recombinant products having no associated structural change. These could formally be interpreted under the HEI model by postulating insertion of ends $e_{1}$ and $e_{2}$ exactly at the non-functional end of either sister element (Figure 2). However, the complementary classes expected under this model, duplicate the right- or left-end elements, are only produced at low frequency (Gray et al., 1996) reducing the likelihood of this model.

The obvious alternative model is one studied above, in which DNA repair is responsible for the exact joining of element ends. The closely linked markers used in this paper show agreement with predictions under the SDSA version of this model. In particular, the distribution of markers in recombinants produced by rejoining of chromosome ends containing $P$ elements is very similar to the distribution from ends that do not contain $P$ elements, suggesting that the same processes apply to the two types of ends.

It therefore follows that dissociation of $P$ element ends rather than insertion into a target site must occur in a substantial fraction of cases. It is interesting to evaluate the likelihood of this outcome in the light of what is known from studies of transposons other than the $P$ element. In the best understood case of transposon insertion, the bacteriophage $M u$, the processes of excision and integration are tightly coupled within a stable 'transpososome' complex (see for example, Chaconas, 1999). Dissociation of element ends would seem unlikely in such a case. However, it appears that the excision and integration processes may be less tightly coupled in mariner elements of Drosophila (Augé-Gouillou et al., 2005) and in transposon Tn10 (Sakai and Kleckner, 1997).

Referring specifically to $P$ elements, although much is now understood about the operation of transposase (Tang et al., 2007), there appears to be no detailed knowledge of the interaction of target sites and transposase. Premature release from any transpososome followed by repair would have no genetical consequences for a normal element and would thus be difficult to detect. And even if the processes are normally tightly coupled in a transpososome structure, it is possible that extra stress is put upon the transpososome by hybrid element ends that are covalently bound to chromosomes rather than to each other. Therefore, the repair process that occurs at such high frequency in the hybrid element case may not be as important in the case of normal $P$ element excision.

The results are, however, important in relating the frequency of hybrid element formation to the recombination frequency for a normal element rather than for the half elements studied here. Recombination is produced by a single element only at the rate of $0.5-1 \%$ (Sved et al., 
1991). Sved and Liang (2006) estimated that sister-strand recombination occurs at a considerably higher rate, of the order of $20 \%$, consistent with recombination rates given by homologous elements (Sved et al., 1991). This difference can be explained if most hybrid element formation events are resolved not by insertion but by repair. It can be seen from Figure 1 (right side) that repair of either element-containing ends or non-element ends leads to sister-strand recombination. The comparison between the low rate of homologous recombination versus the high rate of sister-strand recombination suggests that considerably more than $50 \%$ of hybrid element events might normally be resolved through repair rather than insertion.

\section{Acknowledgements}

We thank Bill Engels and Christine Preston for stocks and advice, and a referee for suggestions for improving the paper. This study was sponsored by grants from the Australian Research Council.

\section{References}

Augé-Gouillou C, Brillet B, Hamelin M-H, Bigot Y (2005). Assembly of the mariner Mos1 Synaptic Complex. Mol Cell Biol 25: 2861-2870.

Beall EL, Rio DC (1997). Drosophila P-element transposase is a novel site-specific endonuclease. Genes Dev 11: 2137-2151.

Chaconas G (1999). Studies on a 'jumping gene machine': higher-order nucleoprotein complexes in $M u$ DNA transposition. Biochem Cell Biol 77: 487-491.

Engels WR, Johnson-Schlitz DM, Eggleston WB, Sved JA (1990). High-frequency $P$ element loss in Drosophila is homologdependent. Cell 62: 515-525.

Gray YHM, Tanaka MM, Sved JA (1996). P element-induced recombination in Drosophila melanogaster: hybrid element insertion. Genetics 144: 1601-1610.
McVey M, Adams M, Staeva-Vieira E, Sekelsky JJ (2004). Evidence for multiple cycles of strand invasion during repair of double-strand gaps in Drosophila. Genetics 167: 699-705.

Nassif NA, Penney J, Pal S, Engels WR, Gloor GB (1994). Efficient copying of nonhomologous sequences from ectopic sites via $P$ element-induced gap repair. Mol Cell Biol 14: $1613-1625$

O'Hare K, Rubin GM (1983). Structure of $P$ transposable elements and their sites of insertion and excision in the Drosophila melanogaster genome. Cell 34: 25-35.

Preston CR, Engels WR (1996). P element-induced male recombination and gene conversion in Drosophila. Genetics 144: 1611-1622.

Preston CR, Flores CC, Engels WR (2006). Differential usage of alternative pathways of double-strand break repair in Drosophila. Genetics 172: 1065-1088.

Preston CR, Sved JA, Engels WR (1996). Flanking duplications and deletions associated with $P$-induced male recombination in Drosophila. Genetics 144: 1623-1638.

Sakai J, Kleckner N (1997). The Tn10 synaptic complex can capture a target DNA only after transposon excision. Cell 89: 205-214.

Sved JA, Blackman LM, Gilchrist AS, Engels WR (1991). High levels of recombination induced by homologous $P$ elements in Drosophila melanogaster. Mol Gen Genet 225: 443-447.

Sved JA, Liang X (2006). Evidence of $P$-element-induced sisterchromatid exchange in a ring- $X$ chromosome in Drosophila, with implication for a high rate of formation of hybrid elements. Genetics 172: 975-979.

Svoboda YHM, Robson MK, Sved JA (1995). P element-induced male recombination can be produced in Drosophila melanogaster by combining end-deficient elements in trans. Genetics 139: 1601-1610.

Tang M, Cecconi C, Bustamante C, Rio DC (2007). Analysis of $P$ element transposase protein-DNA interactions during the early stages of transposition. J Biol Chem 282: 29002-29012.

Weinert BT, Min B, Rio DC (2005). P element excision and repair by non-homologous end joining occurs in both G1 and G2 of the cell cycle. DNA Repair 4: 171-181. 